\title{
LOW HEALTH LITERACY AND EXCESS BODY WEIGHT: A SYSTEMATIC REVIEW
}

\author{
Maria Michou', Demosthenes B. Panagiotakos ${ }^{2}$, Vassiliki Costarelli ${ }^{1}$ \\ ${ }^{1}$ Human Ecology Laboratory, Department of Home Economics and Ecology, Harokopio University, Athens, Greece \\ ${ }^{2}$ Department of Nutrition and Dietetics, Harokopio University, Athens, Greece
}

\section{SUMMARY}

Objective: There is recent evidence that poor health literacy $(\mathrm{HL})$ could be implicated in the aetiology of obesity and could be an important reason behind obese people's inability to encounter difficulties in overcoming obesity issues. The current study reviews the recent scientific evidence investigating the possible link between poor HL levels and excess body weight in adults and children.

Methods: The authors performed a thorough systematic computer-assisted literature search from 1 January 2005 up to 31 May 2017. Only English original studies in healthy people, investigating the relationship between $\mathrm{HL}$ and excess body weight, were included.

Results: Twenty-two studies in total were included in this literature review, 17 studies were conducted in adults and 5 in children. In 17 out of 22 studies reviewed, low HL was significantly associated with increased body mass index, overweight and obesity. In case of children and adolescents, the above association seems to be more consistent compared to adults.

Conclusion: There is good evidence that low levels of $\mathrm{HL}$ are associated with excess body weight, particularly in children. Initiatives to improve health literacy levels could be a useful tool in the management of the obesity epidemic.

Key words: health literacy, obesity, excess body weight, children, adults

Address for correspondence: V. Costarelli, Department of Home Economics and Ecology, Harokopio University of Athens, 70, El. Venizelou Ave, Kallithea, Athens 17671, Greece. E-mail: costarv@hua.gr

https://doi.org/10.21101/cejph.a5172

\section{INTRODUCTION}

Obesity is a widespread problem with major public health implications, however, it has not been yet adequately prevented or treated. In general, poor health literacy is associated with poor health behaviours and outcomes. There is recent evidence, that poor health literacy (HL) could be implicated in the aetiology of obesity and, most importantly, could be an important reason behind obese people's inability to encounter difficulties in overcoming obesity issues (1).

According to the World Health Organization (WHO) health literacy is defined as "The cognitive and social skills which determine the motivation and ability of individuals to gain access to understand and use information in ways which promote and maintain good health" (2). Another term usually used in the literature is the one by the Institute of Medicine of the National Academies, describing HL as "the individuals' capacity to obtain, process and understand basic health information and services needed to make appropriate health decisions" (3). Poor HL simply translates to a lack of understanding one's own wellbeing, of what can be done to improve it, and of what help the healthcare system can offer. In addition to overweight and obesity $(1,4,5)$, low levels of HL have also been associated with several adverse health outcomes such as increased overall mortality (6), higher rates of hospitalization, reduced use of preventive health care services (7), low levels of compliance with prescription medicines (8), difficulty in communication with health professionals
(8), poorer knowledge about illness, and lack of the necessary management skills to control chronic diseases (9). It is important to note that low levels of HL, as it can be expected, are associated with most major chronic degenerative diseases such as hypertension (10), diabetes (11), and depression (12). Health literacy, however, can be improved and therefore is a potentially amenable determinant of health.

\section{Measuring Health Literacy}

There are many different instruments specifically designed to measure HL (13), focusing on different aspects of the definition of HL, hence, making direct comparison between different studies measuring $\mathrm{HL}$ relatively difficult. The most frequently used measures for HL assessment are the Rapid Estimate of Adult Literacy in Medicine (REALM) which measures reading ability and pronunciation (14), the Test of Functional Health Literacy in Adults (TOFHLA) which measures reading, comprehension and numeracy (15), and the Newest Vital Sign (NVS) which measures comprehension and numeracy using the food label of an ice cream (16). Finally, in recent years, a new tool seems to gain ground in HL measurement. This tool is the European Health Literacy Survey Questionnaire (HLS_EU_Q47), which was developed for the European Health Literacy Project in which 8 European countries participated (17). The aim of this paper was to review the most recent scientific evidence linking poor HL levels with excess body weight in adults and children. 


\section{MATERIALS AND METHODS}

\section{Data Sources}

Using a computer-assisted literature search (Medline via PubMed and Scopus), original-research studies that were published in the English language between 1 January 2005 and 31 May 2017 were selected. Combinations of specific keywords were used by the researcher in order to find appropriate articles. The following keywords were used: "health literacy" and "obesity", or "BMI", "body mass index", "body weight", "energy balance", "abdominal obesity", "weight management". In addition, the reference lists of the retrieved articles directed the search to relevant present articles that were not allocated through the searching procedure. Only original studies in the English language have been selected for this review. The initial search resulted in 758 studies both in PubMed and Scopus and we used a hierarchical approach based on title, abstract and full text, in order to assess the relevance of the studies. Exclusion criteria were the use of another language except English, not being an original study such as reviews, case studies etc., and patients with chronic or incurable diseases. In total, 22 studies were included in the current review (17 cross-sectional, 2 randomized control trials (RCT), 1 cohort study, 1 intervention, and 1 mixed method - this study included qualitative and quantitative procedures with seven focus groups and a survey).

\section{RESULTS}

\section{Low Health Literacy and Excess Body Weight}

The studies reviewed in the current paper were separated into studies for adults (over 19 years of age), and study sample size ranged from $n=78$ to $n=162,209$ participants. Twelve studies took place in the United States, 5 in Asia, 3 in Europe, 1 in the United Kingdom, and 1 in Australia. It is important to note that research on HL and obesity in Europe is relatively limited, judging from the relatively scarce scientific literature in the above field published by European authors.

In 17 out of 22 studies reviewed, low HL was significantly associated with increased body mass index, overweight and obesity $(4,5,18-32)$. However, quite a few studies failed to support the above finding. Only one study was found, that linked lower levels of HL with lower BMI, and it was conducted in an elderly population. In the prospective cohort study by Baker et al. conducted in 3,260 elderly people (age > 70 years), levels of adequate HL were $64.2 \%$, marginal HL were $11.2 \%$ and inadequate HL were $24.5 \%$. Participants with inadequate HL were more likely to be underweight $\left(\mathrm{BMI}<18.5 \mathrm{~kg} / \mathrm{m}^{2}\right)$ (33). It is highly likely that, in the elderly, low health literacy translates to higher risk of nutritional deficiencies and poor nutritional status, however, further studies are needed to investigate the above hypothesis.

\section{Studies in Adults}

From 22 studies that were included in this review, 17 assessed HL levels and body weight in adults (Table 1). In 12 out of 17 studies, levels of HL were associated negatively with body weight. According to these studies, as HL levels increased, body weight was reduced and vice versa. However, 5 of these studies did not manage to find any relationship between HL and body weight (34-37).

In all studies, except one, body mass index was calculated for assessing overweight or obesity. In the study where BMI was not calculated, researchers assessed only participants' weight, which is a limitation of the study with respect to assessing obesity (6). Weight and height were measured in 5 studies, whereas in 12 studies, these variables were self-reported. As far as HL measurements are concerned, the most commonly used tools were the NVS and the REALM (4 occasions each), followed by S-TOFHLA (2 occasions), the single self-reported health literacy item: "How confident are you filling out medical forms by yourself?" (2 occasions), and the validated three-item HL screening measure which includes the following 3 questions: "How often do you have someone help you read hospital materials?"; "How confident are you filling out medical forms by yourself?"; "How often do you have problems learning about your medical condition because of difficulty understanding written information?" (2 occasions). Two distinct health literacy dimensions from the 9-dimension Health Literacy Questionnaire (HLQ), the Chinese Citizen Health Literacy Questionnaire, the Health Literacy Management Scale and the European Questionnaire on Literacy for Health (LHS-EU-PT) validated in Portuguese were used once each.

Zoellner et al. wanted to examine whether HL status in employees moderated reach, retention and weight outcomes in a weight loss programme. A two-group cluster randomized controlled trial was implemented which included two 12-month weight loss interventions. The first one was the INCENT programme. Participants had the opportunity to receive daily emails with information about eating and exercise behaviour, they could visit a web site which was targeting weight loss with specific behavioural tools, a kiosk to track weight and their process, and they received some money as a motive for their participation. The other intervention was the LMW programme, which included 4 newsletters and 4 sessions of 1 hour each, using some of the material of the INCENT programme. Each of these procedures occurred once every 3 months. The sample size consisted of 1,460 employees from the USA, $\geq 18$ years old, with a body mass index $>25 \mathrm{~kg} / \mathrm{m}^{2}$. HL significantly moderated the weight loss effects. Specifically, when HL reduced one-unit point, greater weight loss in INCENT compared to LMW was noticed, especially for those with lower baseline HL. Some of the main limitations of the study were the high HL status of the sample and subjective HL measure that was used (4).

In another study by Lanpher et al. a randomized control trial was conducted in 194 overweight and class I obese black women, 25-44 years old, from North Carolina, USA. The aim of the study was to examine differences in socio-demographic, clinical characteristics and weight by HL. Participants randomly assigned either into usual care or into an intervention programme, named Shape Program, which consisted of a 12-month intervention in order to prevent weight gain. Initially, over $50 \%$ of the sample had low HL. Contrary to the results of the previous study, HL did not affect positively weight change after the implementation of the 12-month intervention. This study had several limitations such as the small sample, the aim of the study that was to prevent weight gain, and as a result the variability of weight change is not as that we use to see in weight loss trials, and HL was assessed in 


\begin{tabular}{|c|c|c|c|c|c|}
\hline 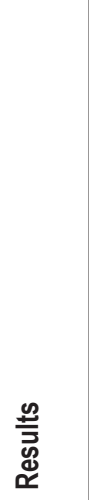 & 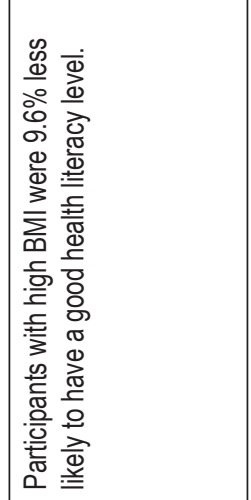 & 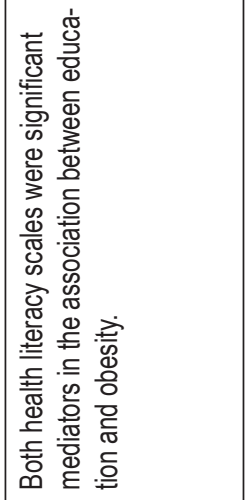 & 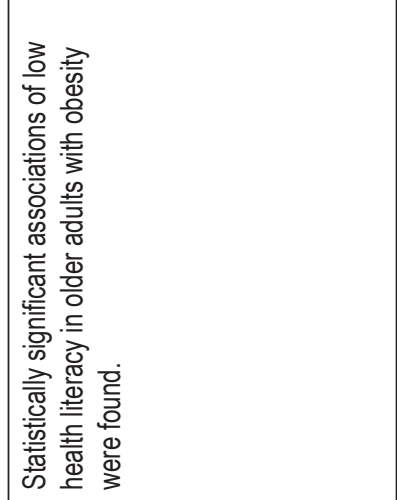 & 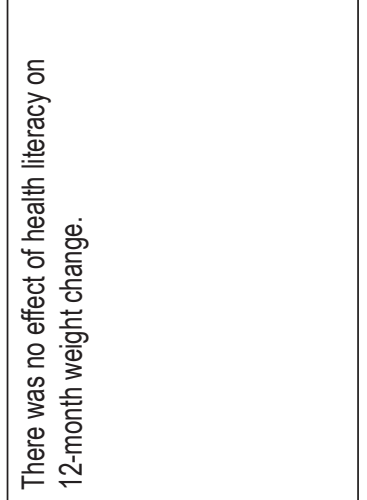 & 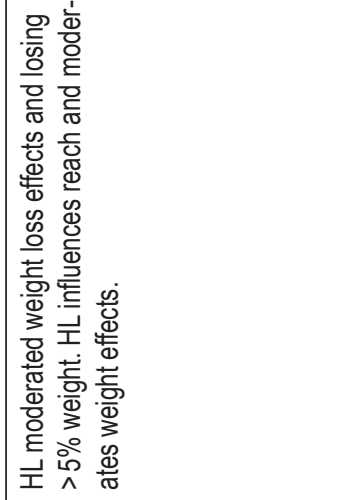 \\
\hline 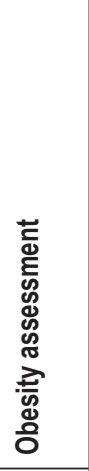 & 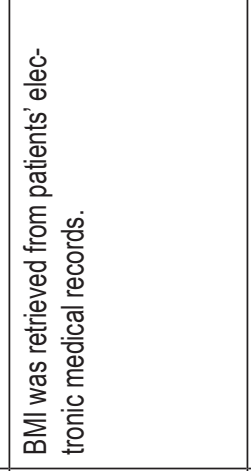 & 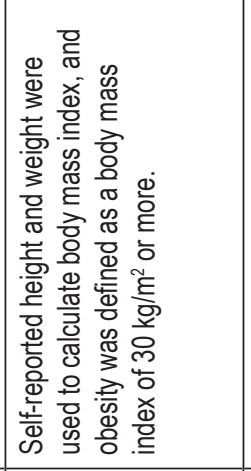 & 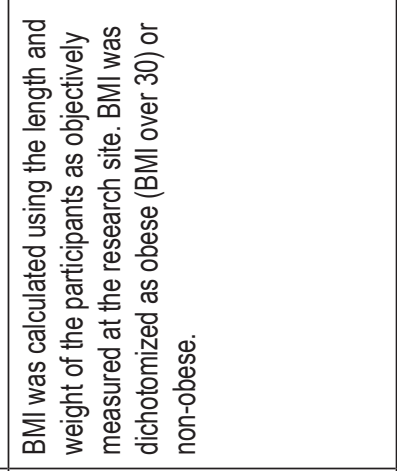 & 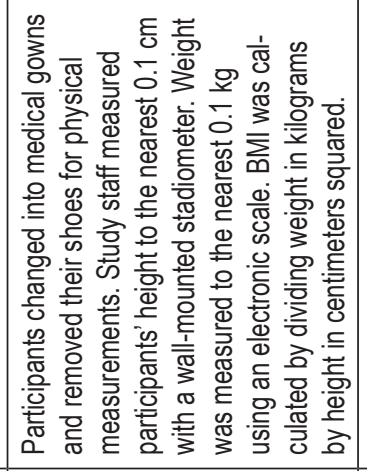 & 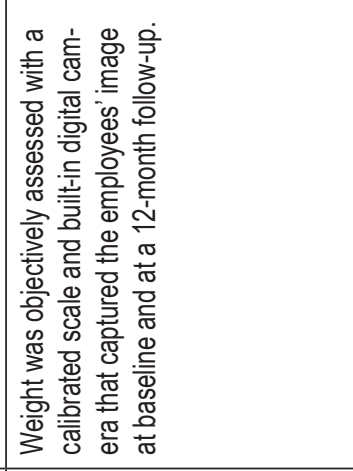 \\
\hline 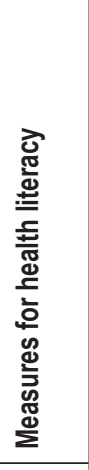 & 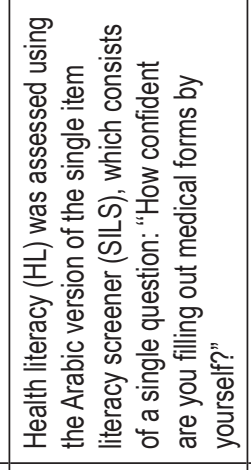 & 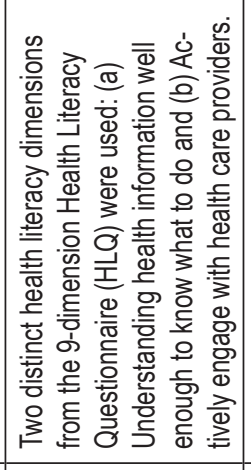 & 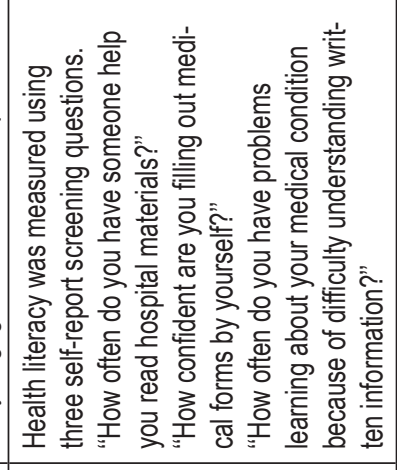 & 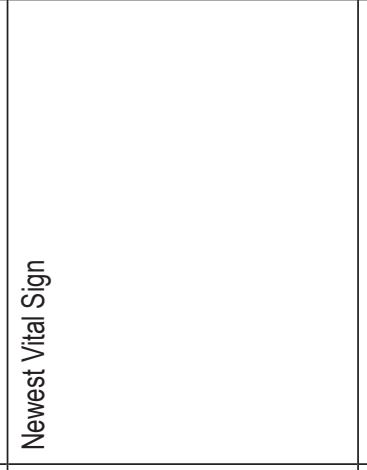 & 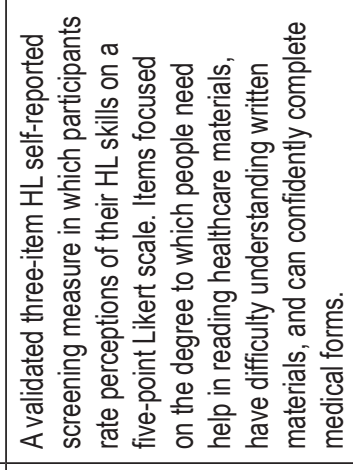 \\
\hline 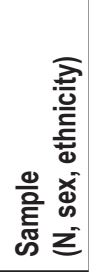 & 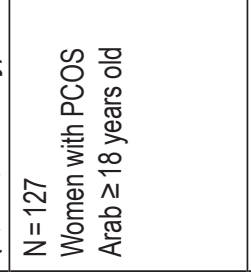 & 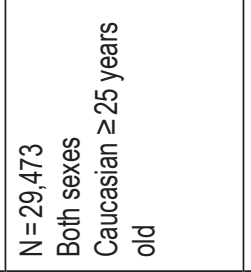 & 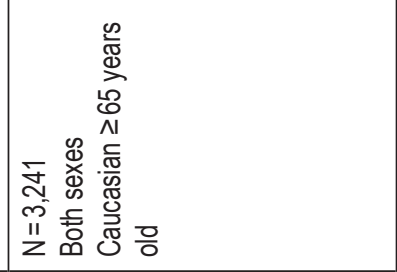 & 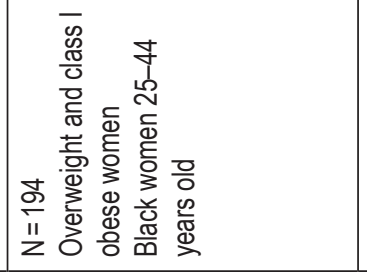 & 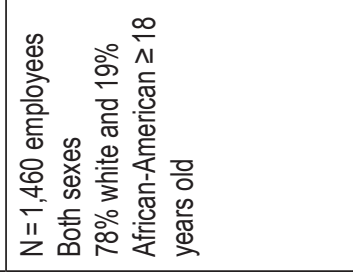 \\
\hline 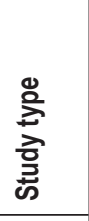 & 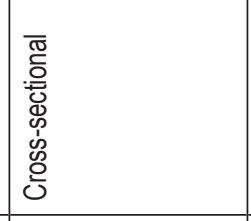 & 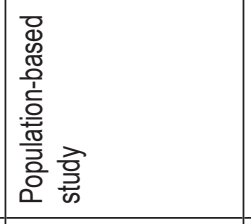 & 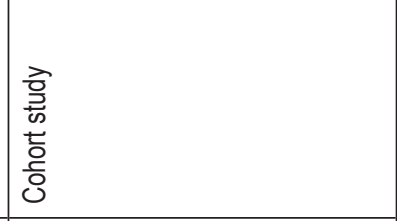 & 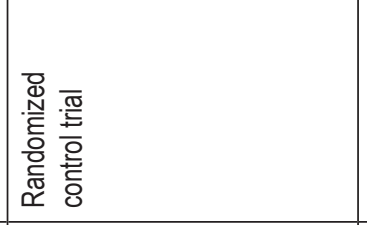 & 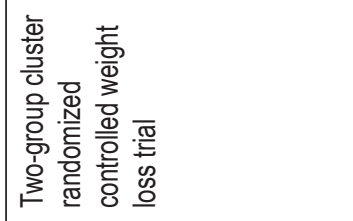 \\
\hline 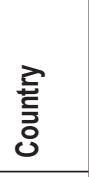 & 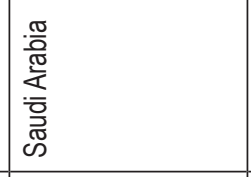 & 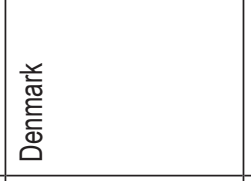 & 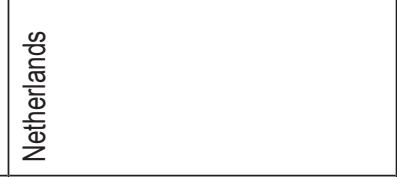 & 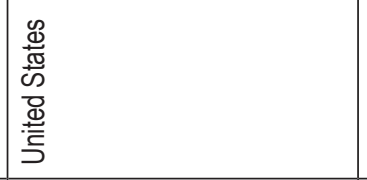 & 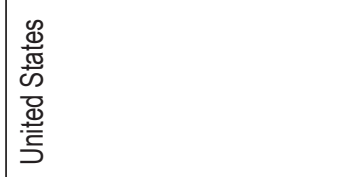 \\
\hline 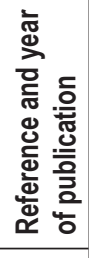 & 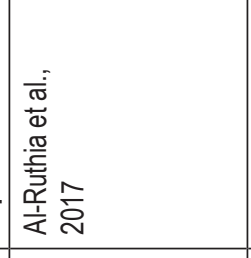 & 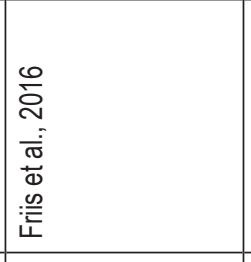 & 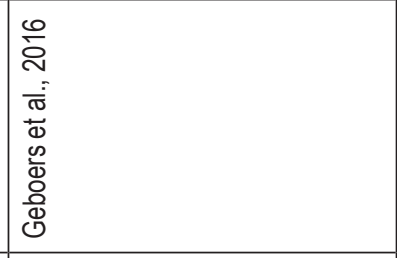 & 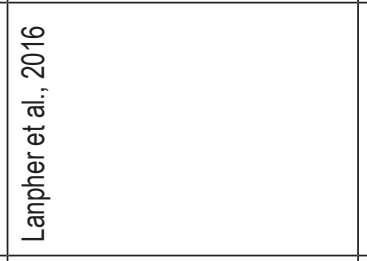 & 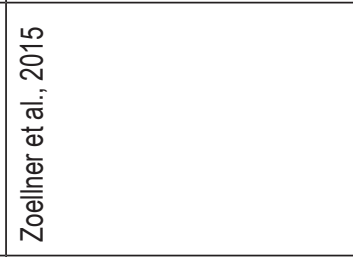 \\
\hline & - & $\sim$ & $m$ & $\nabla$ & مـ \\
\hline
\end{tabular}




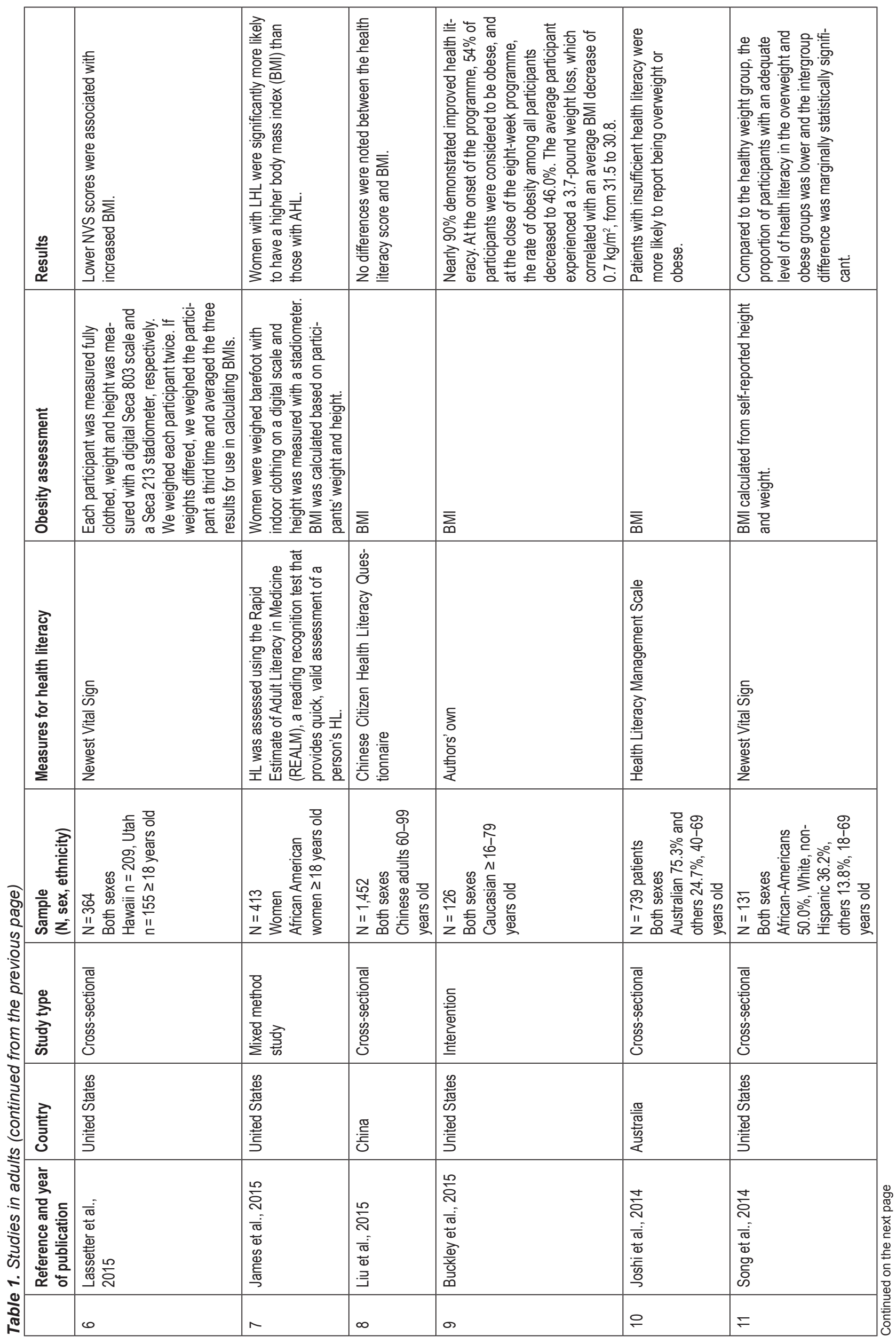




\begin{tabular}{|c|c|c|c|c|c|c|}
\hline $\begin{array}{l}\frac{\mathscr{Q}}{\underline{\bar{z}}} \\
\underline{\underline{g}}\end{array}$ & 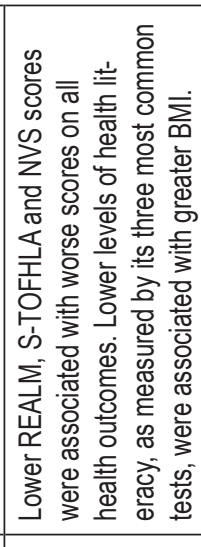 & 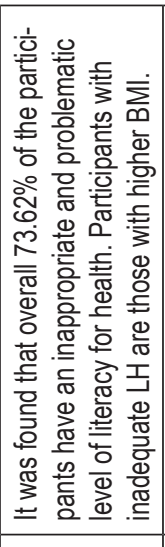 & 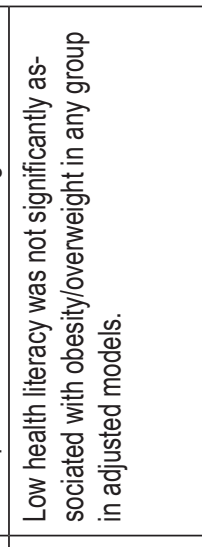 & 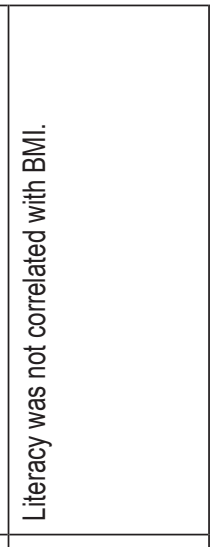 & 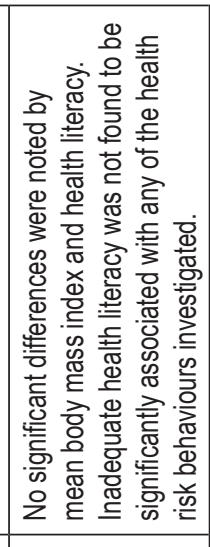 & 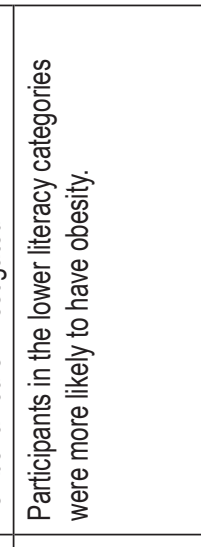 \\
\hline 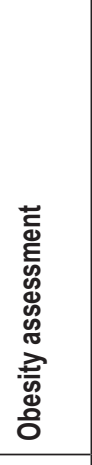 & 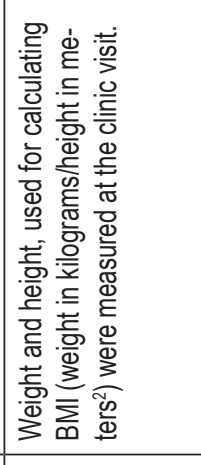 & $\sum_{\text {㐫 }}$ & 商 & $\bar{\Sigma}$ & 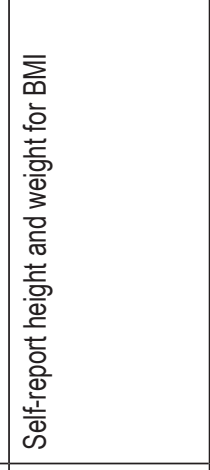 & 商 \\
\hline 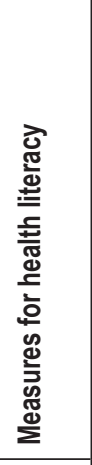 & 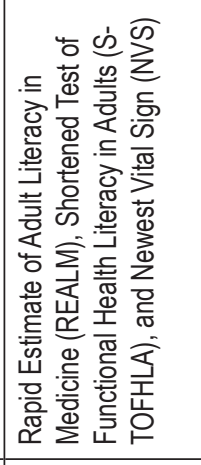 & 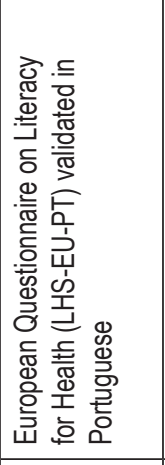 & 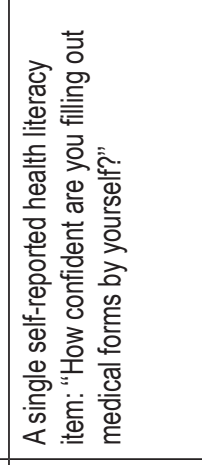 & 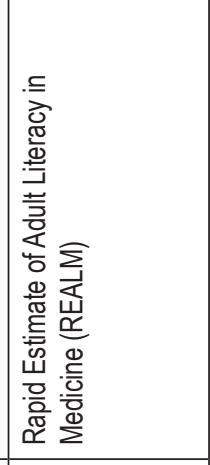 & 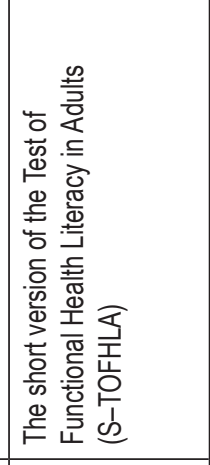 & 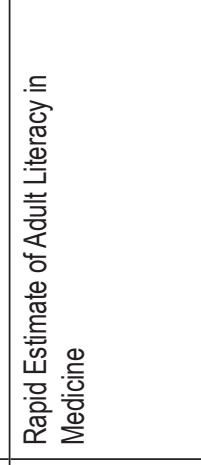 \\
\hline 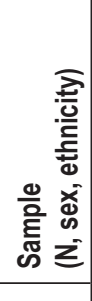 & 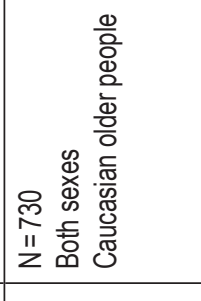 & 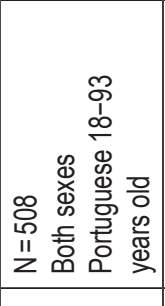 & 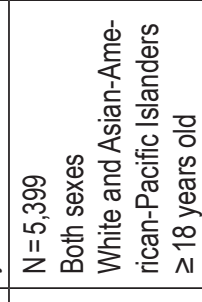 & 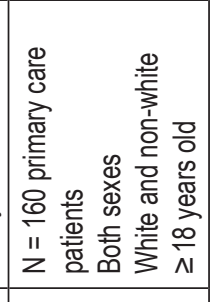 & 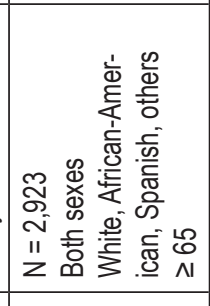 & 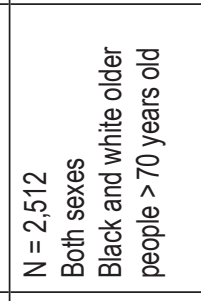 \\
\hline 总 & 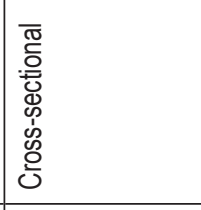 & 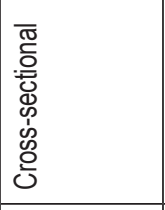 & 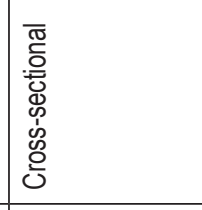 & 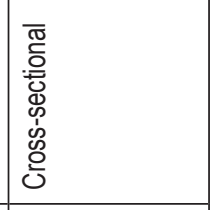 & 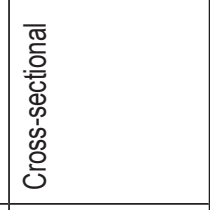 & 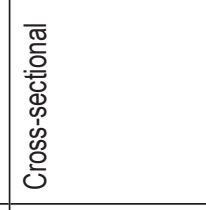 \\
\hline 吾 & 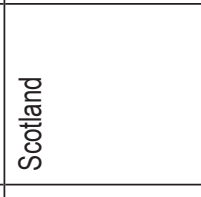 & \begin{tabular}{|l} 
喾 \\
言
\end{tabular} & 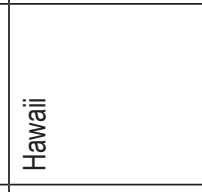 & 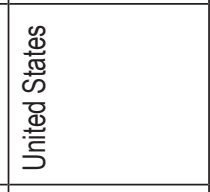 & 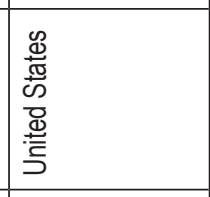 & 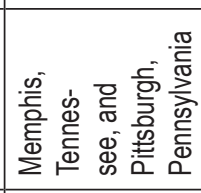 \\
\hline 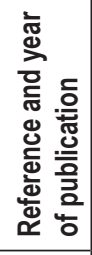 & 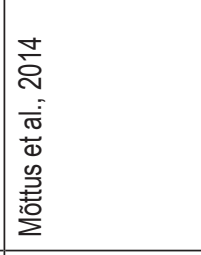 & 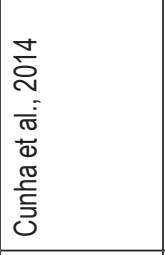 & 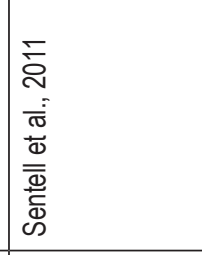 & 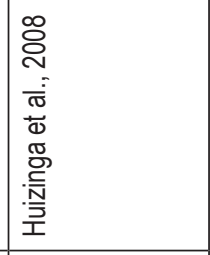 & 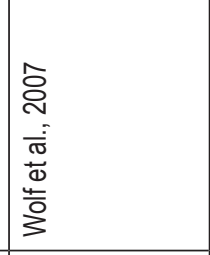 & 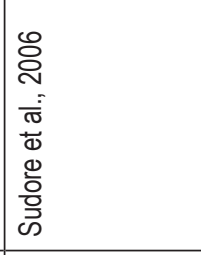 \\
\hline & $\simeq$ & $\cong$ & 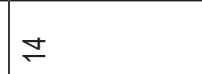 & $\stackrel{2}{\circ}$ & 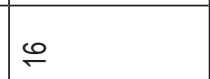 & $\approx$ \\
\hline
\end{tabular}




\begin{tabular}{|c|c|c|c|c|c|}
\hline 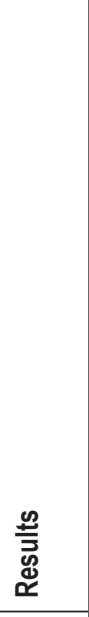 & 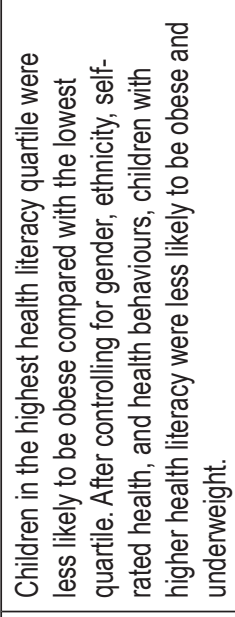 & 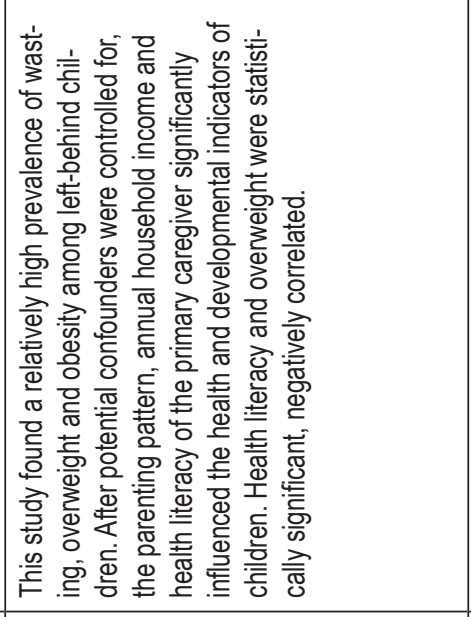 & 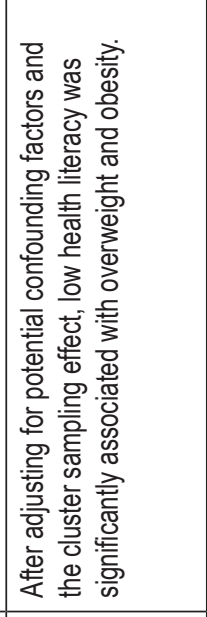 & 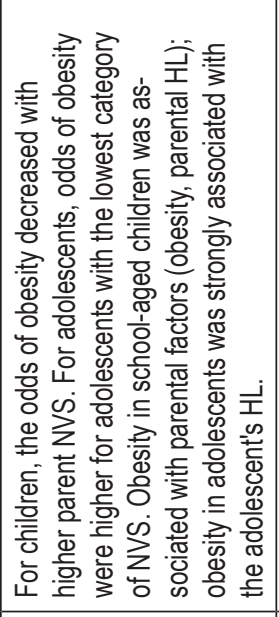 & 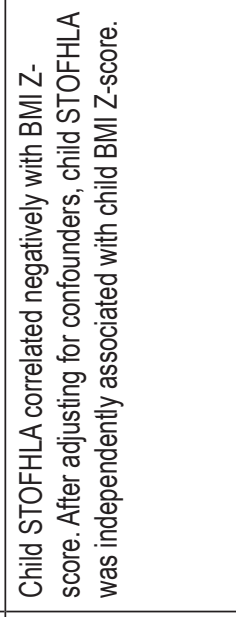 \\
\hline 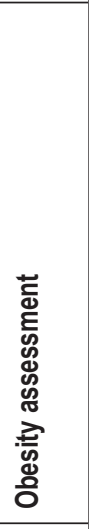 & 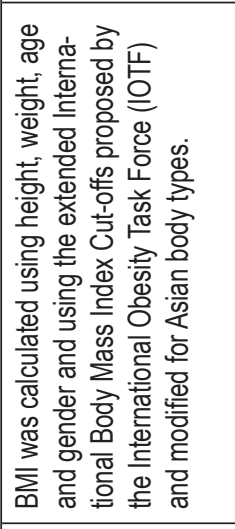 & 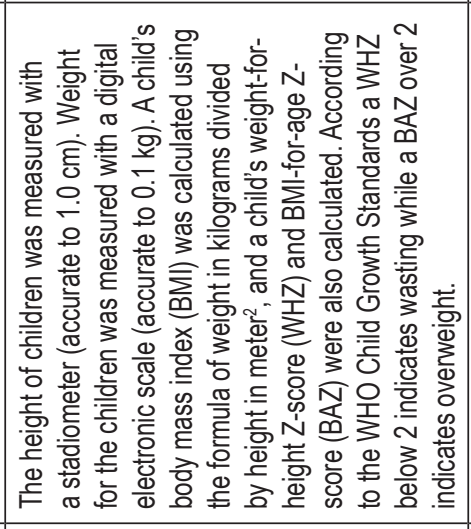 & 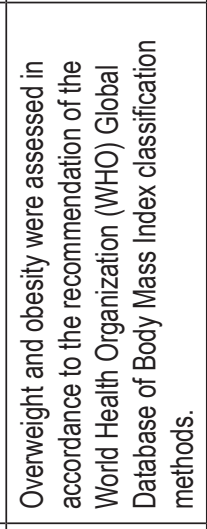 & 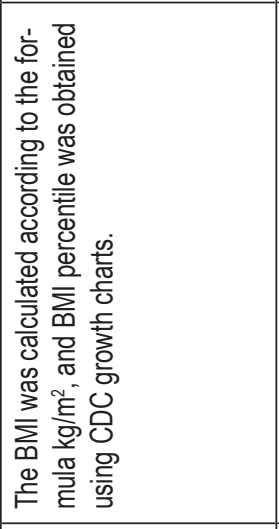 & 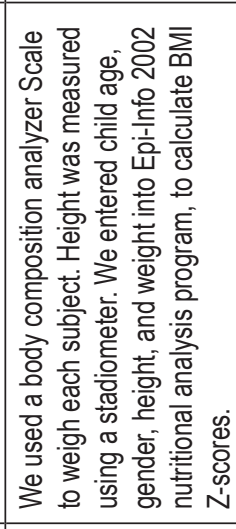 \\
\hline 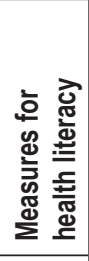 & 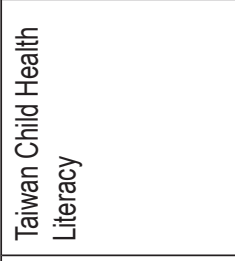 & 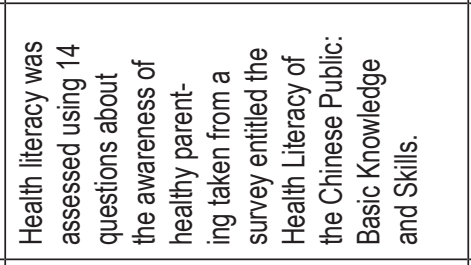 & 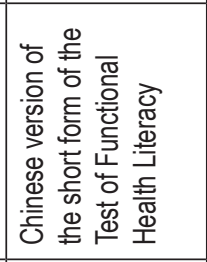 & 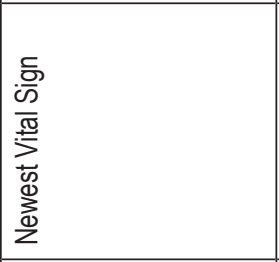 & 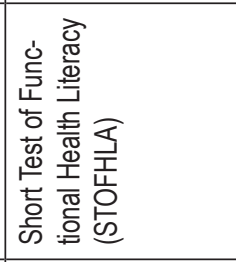 \\
\hline 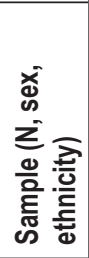 & 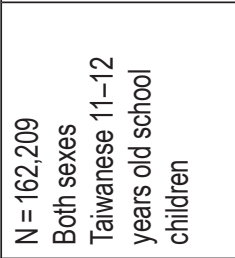 & 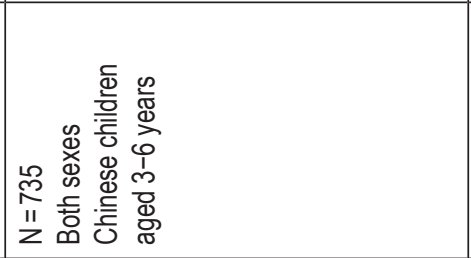 & 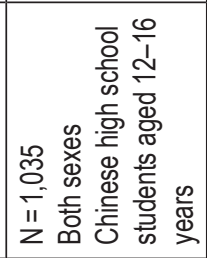 & 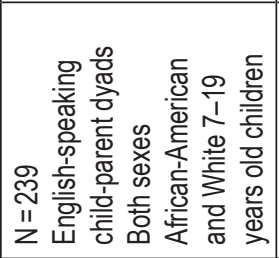 & 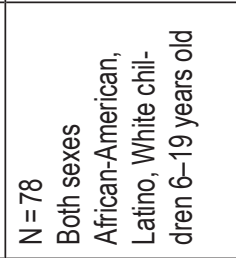 \\
\hline 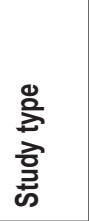 & 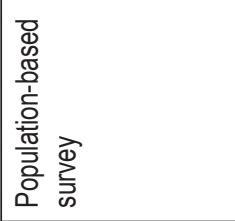 & 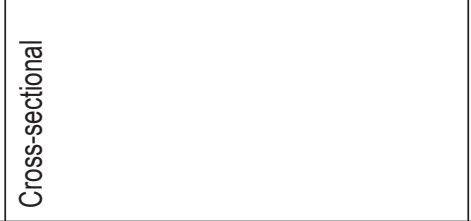 & 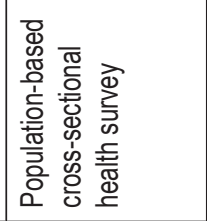 & 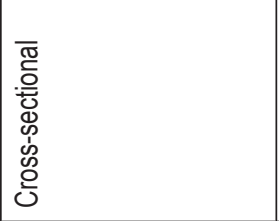 & 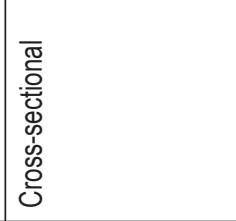 \\
\hline 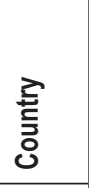 & 旅 & 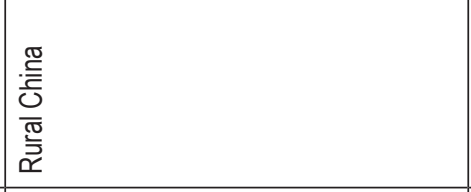 & 离 & 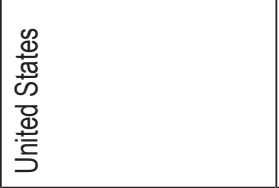 & 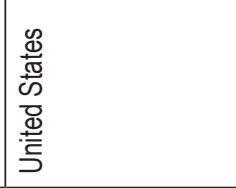 \\
\hline 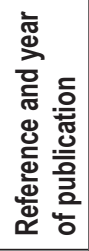 & 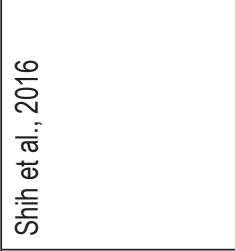 & 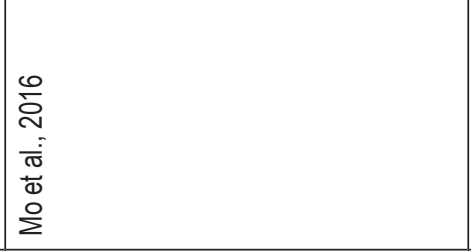 & 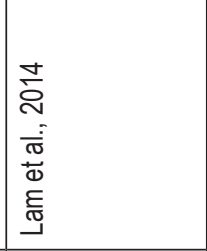 & 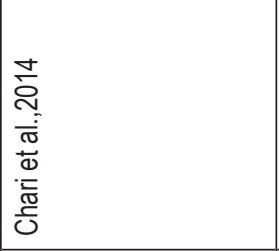 & 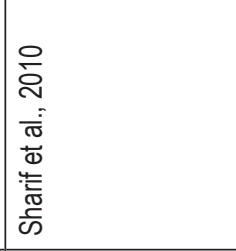 \\
\hline & - & $\sim$ & m & $\theta$ & ما \\
\hline
\end{tabular}


the 18 months visit only by NVS, a tool that may not be sensitive to middle levels of HL (34).

It is worth mentioning that a study which aimed at examining the relationship between BMI and HL among women with polycystic ovary syndrome (PCOS) was included in this review. This very recent cross-sectional study, published in 2017 by Al-Ruthia et al., included women of mean age 27.40 years $( \pm 5,35)$. HL was assessed by the Arabic version of the single item literacy screener (SILS), which consists of a single question: "How confident are you filling out medical forms by yourself?". After controlling for confounding factors such as age, education etc., PCOS patients with higher BMI were almost 10\% less likely to have good HL compared to others with lower BMI. The limitations of this study were the use of only one HL item and that the results cannot be generalized due to the study design (18).

\section{Studies in Children and Adolescents}

Five from 22 studies in this review assessed HL levels and body weight in children and adolescents up to 19 years of age (Table 2) (28-32). In case of children and adolescents, the possible association between low health literacy and excess body weight seems to be more consistent. More specifically, all the studies conducted in this age group and included in this review found a significant association of low HL levels with overweight and obesity. In 2 studies, parental HL was assessed in order to investigate if there is a link with child's body weight, and in the remaining 3 studies, child's HL was assessed. In all 5 studies, weight and height were measured and body mass index was calculated for assessing overweight or obesity. The instruments to assess HL differed a lot. The STOFHLA was used in 2 studies and NVS, Taiwan Child Health Literacy and 14 questions about the awareness of healthy parenting taken from a survey entitled the Health Literacy of the Chinese Public: Basic Knowledge and Skills, were used once each.

An interesting population-based study occurred in Taiwan by Shih et al. The sample was particularly large $(n=162,209)$ and consisted of Taiwanese 11-12 years old school children. HL was assessed by the Taiwan Child Health Literacy measure and BMI was calculated using height, weight, age and gender in accordance with the extended International Body Mass Index Cut off points, proposed by the International Obesity Task Force (IOTF) and modified for Asian body types. Results showed that children with higher HL were less likely to be obese and underweight (28).

\section{DISCUSSION}

The purpose of the current literature review was to investigate the evidence linking poor HL levels with excess body weight in adults and children. The majority of the studies included in this review indicate that low levels of health literacy are associated with excess body weight. This association seems to be more profound in case of children and adolescents in comparison to adults.

In a similar paper by Faruqi et al., which systematically reviewed primary health care level interventions targeting health literacy, in order to assess their effect on weight loss, the majority of the studies reviewed (11 out of 13 studies) resulted in a significant reduction in weight and/or BMI, in at least one follow-up visit (1). Another systematic review by Sansom-Daly et al., which included studies for health literacy in adolescents and young adults, also concluded that poorer health literacy was associated with some adverse health outcomes, one of which was obesity (38).

Low levels of SES are associated with obesity in developed countries, especially in women (39). In the current review, in 9 out of 22 studies in total, there was a significant negative correlation between HL levels and socioeconomic status (SES). More specifically, SES was examined and the results indicated that low levels of HL were more likely to occur in participants with lower education, lower income and lower occupational social class. However, the interrelationships between SES, HL and obesity, were not examined in these studies. As for the race and ethnicity, Caucasian participants were more likely to have higher levels of HL than the remaining groups (5, 24-27, 31, 34-36).

There is evidence that HL and excess body weight are negatively associated, however, there are discrepancies in the findings of the studies reviewed in this paper, particularly in the adult population. This could partly be attributed to a number of limitations of the studies with respect to the methodology, the sampling method and different methods used to assess HL. A major problem, that the concept and measurement of health literacy has, is the heterogeneity of HL measurements used. Health literacy, due to its multidimensional construct, has many definitions and theoretical frameworks and the result is the lack of a gold standard measure. More than 50 tools for measuring HL exist (13), but each of them measures only some aspects of HL. Also, every tool seems to have psychometric weaknesses. For example, when some of these tools, such as the REALM, TOFHLA and NVS, are given to the participants at the same time, the results are different in terms of HL levels (40). This renders the comparison between the studies' results difficult. Moreover, another important limitation of this review is that in more than half of the studies participants' weight and height were self-reported.

\section{CONCLUSIONS}

There is evidence that low HL could be implicated in the aetiology of obesity and could be an important reason behind obese people's inability to succeed in loosing excess body weight or maintaining normal weight status. Improving health and nutrition literacy could empower people to effectively manage long-term health conditions and reduce the burden on health and social care services. More specifically, increasing health literacy levels might be a useful tool in better managing the obesity epidemic in Europe and worldwide.

More studies, particularly in Europe, are needed to better elucidate the possible interrelationships between HL levels and excess body weight in different populations in order to better understand the role of increasing health literacy in maintain healthy lifestyle factors and good health.

\section{Funding}

This research has been financially supported by the General Secretariat for Research and Technology (GSRT) and the Hellenic Foundation for Research and Innovation (HFRI) (Scholarship Code: 949). 


\section{Conflict of Interests}

None declared

\section{REFERENCES}

1. Faruqi N, Spooner C, Joshi C, Lloyd J, Dennis S, Stocks N, et al. Primary health care-level interventions targeting health literacy and their effect on weight loss: a systematic review. BMC Obesity. 2015;2:6. doi: 10.1186/ s40608-015-0035-7.

2. World Health Organization. Health promotion. Track 2: health literacy and health behaviour [Internet]. Geneva: WHO; 2018 [cited 2018 Sep 7] Available from: http://www.who.int/healthpromotion/conferences/7gchp/ track $2 / \mathrm{en} /$.

3. Institute of Medicine. Health literacy: a prescription to end confusion. Washington DC: The National Academies; 2004.

4. Zoellner J, You W, Almeida F, Blackman KC, Harden S, Glasgow RE, et al. The Influence of health literacy on reach, retention, and success in a worksite weight loss program. Am J Health Promot. 2016;30(4):279-82.

5. Lassetter JH, Clark L, Morgan SE, Brown LB, VanServellen G, Duncan $\mathrm{K}$, et al. Health literacy and obesity among native Hawaiian and Pacific Islanders in the United States. Public Health Nurs. 2015;32(1):15-23.

6. Peterson PN, Shetterly SM, Clarke CL, Allen LA, Matlock DD, Magid DJ, et al. Low health literacy is associated with increased risk of mortality in patients with heart failure. Circulation. 2009;120(18):S749.

7. Scott TL, Gazmararian JA, Williams MV, Baker DW. Health literacy and preventive health care use among medicare enrollees in a managed care organization. Med Care. 2002;40(5):395-404.

8. Schillinger D, Bindman A, Wang F, Stewart A, Piette J. Functional health literacy and the quality of physician-patient communication among diabetes patients. Patient Educ Couns. 2004;52(3):315-23.

9. Schillinger D, Grumbach K, Piette J, Wang F, Osmond D, Daher C, et al. Association of health literacy with diabetes outcomes. JAMA. 2002;288(4):475-82.

10. McNaughton CD, Kripalani S, Cawthon C, Mion LC, Wallston KA, Roumie CL. Association of health literacy with elevated blood pressure: a cohort study of hospitalized patients. Med Care. 2014;52(4):346-53.

11. Cavanaugh KL. Health literacy in diabetes care: explanation, evidence and equipment. Diabetes Manag (Lond). 2011;1(2):191-9.

12. Lincoln A, Paasche-Orlow MK, Cheng DM, Lloyd-Travaglini C, Caruso C, Saitz R, et al. Impact of health literacy on depressive symptoms and mental health-related quality of life among adults with addiction. J Gen Intern Med. 2006;21(8):818-22.

13. Haun JN, Valerio MA, McCormack LA, Sørensen K, Paasche-Orlow MK. Health literacy measurement: an inventory and descriptive summary of 51 instruments. J Health Commun. 2014;19(2):302-33.

14. Davis TC, Crouch M, Long S. Rapid estimate of adult literacy in medicine. Fam Med. 1993;25(6):391-5.

15. Parker R, Baker D, Williams M, Nurss J. The test of functional health literacy in adults: a new instrument for measuring patients' literacy skills J Gen Intern Med. 1995;10(10):537-41.

16. Weiss BD, Mays MZ, Martz W, Castro KM, DeWalt DA, Pignone MP, et al. Quick assessment of literacy in primary care: the newest vital sign. Ann Fam Med. 2005;3(6):514-22

17. Sørensen K, Van den Broucke S, Pelikan JM, Fullam J, Doyle G, Slonska $Z$, et al. Measuring health literacy in populations: illuminating the design and development process of the European Health Literacy Survey Questionnaire (HLS-EU-Q). BMC Public Health. 2013;13:948. doi: 10.1186/1471-2458-13-948.

18. Al-Ruthia YS, Balkhi B, AlGhadeer S, Mansy W, AlSanawi H, AlGasem $\mathrm{R}$, et al. Relationship between health literacy and body mass index among Arab women with polycystic ovary syndrome. Saudi Pharm J. 2017;25(7):1015-18.

19. Friis K, Lasgaard M, Rowlands G, Osborne RH, Maindal HT. Health literacy mediates the relationship between educational attainment and health behavior: a Danish population-based study. J Health Commun. 2016;21 Suppl 2:54-60.

20. Geboers B, Reijneveld SA, Jansen CJ, de Winter AF. Health literacy is associated with health behaviors and social factors among older adults: results from the lifelines cohort study. J Health Commun. 2016;21 Suppl 2:45-53.
21. James DS, Harville C, Efunbumi O, Martin MY. Health literacy issues surrounding weight management among African American women: a mixed methods study. J Hum Nutr Diet. 2015;28(2):41-9.

22. Buckley J, Yekta S, Joseph V, Johnson H, Oliverio S, De Groot AS. Vida Sana: a lifestyle intervention for uninsured, predominantly Spanishspeaking immigrants improves metabolic syndrome indicators. J Commun Health. 2015;40(1):116-23.

23. Joshi C, Jayasinghe UW, Parker S, Del Mar C, Russell G, Lloyd J, et al. Does health literacy affect patients' receipt of preventative primary care? A multilevel analysis. BMC Family Pract. 2014;15:171. doi: 10.1186/ s12875-014-0171-z.

24. Song HJ, Grutzmacher SK, Kostenko J. Personal weight status classification and health literacy among Supplemental Nutrition Assistance Program (SNAP) participants. J Commun Health. 2014;39(3):446-53.

25. Mõttus R, Johnson W, Murray C, Wolf MS, Starr JM, Deary IJ. Towards understanding the links between health literacy and physical health. Health Psychol. 2014;33(2):164-73.

26. Cunha M, Gaspar R, Fonseca S, Almeida D, Silva M, Nunes L. Implications of literacy for health for body mass index. Aten Primaria. 2014;46(1):180-6.

27. Sudore RL, Mehta KM, Simonsick EM, Harris TB, Newman AB, Satterfield S, et al. Limited literacy in older people and disparities in health and healthcare access. J Am Geriatr Soc. 2006;54(5):770-6.

28. Shih SF, Liu CH, Liao LL, Osborne RH. Health literacy and the determinants of obesity: a population-based survey of sixth grade school children in Taiwan. BMC Public Health. 2016;16:280. doi: 10.1186/s12889-0162879-2.

29. Mo X, Xu L, Luo H, Wang X, Zhang F, Gai Tobe R. Do different parenting patterns impact the health and physical growth of 'left-behind' preschoolaged children? A cross-sectional study in rural China. Eur J Public Health. 2016;26(1):18-23.

30. Lam LT, Yang L. Is low health literacy associated with overweight and obesity in adolescents: an epidemiology study in a 12-16 years old population, Nanning, China, 2012. Arch Public Health. 2014;72(1):11. doi: 10.1186/2049-3258-72-11

31. Chari R, Warsh J, Ketterer T, Hossain J, Sharif I. Association between health literacy and child and adolescent obesity. Patient Educ Couns. 2014;94(1):61-6.

32. Sharif I, Blank AE. Relationship between child health literacy and Body Mass Index in overweight children. Patient Educ Couns. 2010;79(1):43-8.

33. Baker DW, Wolf MS, Feinglass J, Thompson JA, Gazmararian JA, Huang J. Health literacy and mortality among elderly persons. Arch Intern Med. 2007;167(14):1503-9.

34. Lanpher MG, Askew S, Bennett GG. Health literacy and weight change in a digital Health intervention for women: a randomized controlled trial in primary care practice. J Health Commun. 2016;21(1):34-42.

35. Liu YB, Liu L, Li YF, Chen YL. Relationship between health literacy, health-related behaviors and health status: a survey of elderly Chinese. Int J Environ Res Public Health. 2015;12(8):9714-25.

36. Sentell T, Baker KK, Onaka A, Braun K. Low health literacy and poor health status in Asian Americans and Pacific Islanders in Hawai'i. J Health Commun. 2011;16(3):279-94.

37. Wolf MS, Gazmararian JA, Baker DW. Health literacy and health risk behaviors among older adults. Am J Prev Med. 2007;32(1):19-24.

38. Sansom-Daly UM, Lin M, Robertson EG, Wakefield CE, McGill BC, Girgis A, et al. Health literacy in adolescents and young adults: an updated review. J Adolesc Young Adult Oncol. 2016;5(2):106-18.

39. Newton S, Braithwaite D, Akinyemiju TF. Socio-economic status over the life course and obesity: systematic review and meta-analysis. PLoS ONE. 2017;12(5):e0177151. doi: 10.1371/journal.pone.0177151.

40. Osborne RH, Batterham RW, Elsworth GR, Hawkins M, Buchbinder R. The grounded psychometric development and initial validation of the Health Literacy Questionnaire (HLQ). BMC Public Health. 2013;13:658. doi: 10.1186/1471-2458-13-658. 